\section{REFLIST: Programs to compile a reference list from word processor text}

\author{
NEIL R. SHAPIRO and ROBERT A. ROSELLINI \\ State University of New York at Albany \\ Albany, New York 12222
}

With the decreasing price of microcomputers, their use as word processors to prepare manuscripts is on the increase. However, the author is still left with the tedious and error prone task of assembling a reference list of citations in the manuscript. This involves a manual search of the text for citations, alphabetizing the citations, and completing a reference for each item in the list. However, alphabetization is easily accomplished via a computerized sort, and since the same references appear in many different manuscripts, compilation of the reference list can be expedited by a computerized search of a master reference file. It is desirable to have the computer assemble a citation list and create a reference list automatically. Such a facility not only saves time but also insures greater accuracy of citations in a reference list.

The programs described here, when used in conjunction with a word processor program such as SCRIPSIT, perform these functions with little user intervention.

Program Description. The programs are written according to the syntax of Radio Shack Level II disk BASIC 1.1 and their editor-assembler. The first of these, REFLIST1, searches the text of a manuscript held in memory for the occurrence of citations following the American Psychological Association (APA) formatthat is, a name or list of names followed by a date. The search is implemented by an assembly language routine that is supervised by the BASIC program. Upon completion of the search, a disk file is created which contains all citations in the order in which they appear in the text. This file includes multiple references to the same work but does not include citations using "et al." to indicate repetition.

A second BASIC program, REFLIST2, serves three general functions: (1) It allows the user to edit the citation list created by REFLIST 1 so that errors in the text citations do not influence the alphabetization of the list; (2) it alphabetizes the corrected list, deleting multiple citations to the same source; (3) after creating a list of unique references, REFLIST2 searches a master reference file for each reference. These functions are described in more detail below.

The editing feature allows the user to delete or modify references contained in the citation list. For example, if authors' names are misspelled or cited out of order, the reference can be corrected at this point. In

This work was supported by NSF Grant BNS-7820678 to the second author. addition, references can be inserted during editing if, for example, one notes that a reference was left out that is to be added to the text.

When editing is completed, the reference list is alphabetized. The alphabetized list can be quite helpful in finding errors that may exist in citation format in the original text. Since citations are compared character by character, the program considers references to the same work as distinct if the format of citation varies in the text. Thus, inconsistencies within the manuscript are easily found.

The compilation of the complete reference list is interactive. The "target" citation is first printed; then the master reference list is searched for a match to each author's last name. When a match occurs, the user is asked to verify whether the match is correct. If so, the reference is included in the final list. If the match is incorrect, the search is continued. If the reference does not exist in the master reference file, the user has the option of updating the master reference file or continuing with the compilation of the reference list, excluding that reference. When compilation is complete, the word processor program should be used to perform any lastminute editing of the completed reference section and to print it according to the desired format for page length, margins, etc.

Program Requirements. These programs are written for the TRS-80 Model 1 or 3 , with $48 \mathrm{k}$ or $64 \mathrm{k}$ of memory and at least one disk drive. They are specifically written to work with Radio Shack's word processing system, SCRIPSIT.' The instruction manual provides information that should permit the user to modify the program for other word processors. In most cases, only minor modifications to the BASIC program are involved.

In the distributed form, the programs require the NEWDOS80 disk operating system. ${ }^{2}$ Incompatibility with other disk operating systems, such as the standard TRSDOS, stems from the execution of DOS commands from BASIC, including the execution of a DOS chain file. The programs can be modified for use with disk operating systems not offering these features; however, more manual operations (e.g., file transfers, program calls) are necessary.

Availability. A program listing, an operations manual with example runs of the programs, and a copy of the programs on a minifloppy diskette can be obtained from the authors at cost.

\section{NOTES}

1. The SCRIPSIT word processor program is available from Radio Shack.

2. The NEWDOS80 disk operating system is available from Apparat, Inc., 4401 South Tamarac Parkway, Denver, Colorado 80237.

(Accepted for publication November 17, 1981.) 